\title{
SMALL SIGNAL MODEL FOR PV FED BUCK CONVERTER IN CONTINUOUS AND DISCONTINUOUS CONDUCTION MODES
}

\author{
Pratibha Katti \\ M.Tech, EEE Department \\ B.M.S College of Engineering, \\ Bengaluru,Karnataka,India
}

\author{
Dr Pradeepa $S$ \\ Professor, EEE Department \\ B.M.S College of Engineering \\ Bengaluru,karnataka,India
}

\author{
Dr Arjun M \\ Assistant Professor,EEE \\ Department \\ B.M.S College of Engineering \\ Bengaluru,Karnataka,India
}

\begin{abstract}
Small signal modelling is of great significance in power electronics and is a common analysis technique used to approximate the behavior of systems that evince extremely nonlinearity characteristics. They aid to ingress into stabilizing and in tuning the controllers as well. Over many power electronic converters's small signal modelling have been interpreted throughout literature. Though, in few applications such as photovoltaic systems necessitate the introducing with capacitor located close to photovoltaic panels terminals. The above capacitor will be linked amid photovoltaic panel and power converter's input side, is well-defined as an input capacitor. In recent investigation, it is established that impact of introducing input capacitor upon system stability was not dependent for PV fed boost converter. Yet, the result of input capacitor addition for all other power converters is not derived. In this paper, we develop small signal models under both CCM and DCM working conditions for photovoltaic fed non - ideal buck converter. The presumed load can be virtuously resistive and therefore it even remains acceptable for any functional loads. It is found that even for buck converter the stability of MPPT's system is sovereign of input capacitance in both operating conditions and also it proves that MPPT system is open loop robust.
\end{abstract}

Keywords - Continuous Conduction Mode (CCM), Discontinuous Conduction Mode (DCM), Photovoltaic (PV), maximum power point tracking (MPPT)

\section{INTRODUCTION}

Photovoltaic systems are among the most fragile of all the renewable sources of energy that are frequently classified as low - efficiency types of energy conversion. The current research therefore concentrates on enhancing the energy harnessing reliability by relying primarily on MPPT. The maximum power point tracking (MPPT) strategies typically include regulating the voltage and current of the panels utilizing power converter relying upon insolation levels and temperature [1] [2]. Every other system intended for control necessitates feedback. The feedback system is designed to meet stringent conditions like stability, including properties like time settling, overshooting [3].We require dynamic model for developing a robust feedback system. Such dynamics incorporated by parameters like inductors, capacitors is analyzed well with aid of small signal modelling. In Literature we can widely see models for stability of voltage control as well as current control methods.

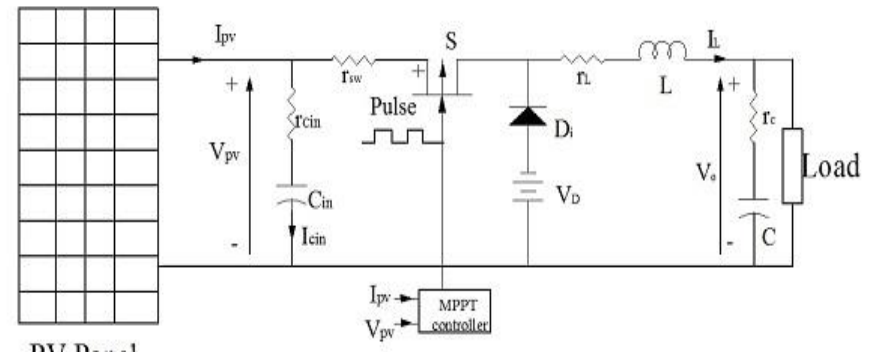

PV Panel

Fig. 1 schematic diagram for PV powered buck converter over MPPT mode connected to load [2]

Nevertheless, stability models are rarely addressed for photovoltaic powered plant with an ancillary input capacitor. The analysis contemplates input capacitor through modelling. Nonetheless, there is no clear analysis of the influence for input capacitor upon stability. This is still uncertain if the input capacitor will have similar impact upon stability for other power converters or else may be it is unique to the converter. It is found that majority of models presume the converter is functioning in CCM. Nevertheless, PV systems are sometimes found to enter into DCM [4] Here are few points to remember from all the above discussions. Stability models are rarely addressed, specifically for PV fed system. The impact of input capacitance upon system stability is being addressed solely in recent studies for boost converter functioning in both CCM and DCM [6]. Therefore it is not investigated the impact of input capacitance upon system stability for photovoltaic fed systems for other power converters. Considering the subject under review, this paper's findings are

- It aims at developing a stability models by comprehensive derivations for a non- ideal buck 
converter functioning under both CCM and DCM modes.

- Furthermore it demonstrates engrossing outcomes for both the modes like pole - zero cancellations contributing to pertinent result.

\section{SMALl Signal MODEL}

Fig.1 displays the schematic diagram, where PV feeds non ideal buck converter connected to a load. Here load assumed is Resistive load, it can also be assumed as any other complex load. $V_{c_{\text {in }}}$ represents voltage across the capacitor located to input side. $V_{p v}$ represents the voltage across photovoltaic panels and $V_{c}$ represents the votage across capacitor at the output side. $I_{L}$ represents current flowing through the inductor and $I_{p v}$ represents current flowing through the PV terminals. $C_{i n}$ represents the capacitor which is connected amid the PV panels and buck converter called input capacitor. $L$ represents inductor, $C$ represents capacitor situated at output side of the buck converter. $V_{o}$ represents the output voltage of the non - ideal buck converter. Small signal modeling is obtained by considering the effective series resistance (esr) of the circuit parameters. Here $r_{c}, r_{c_{i n}}, r_{s w}, r_{L}$ represents the non-idealities of parameters such as output capacitor, input capacitor, switch, and inductor correspondingly. $V_{D}$ represents the reduction in voltage in diode $D_{i}$.

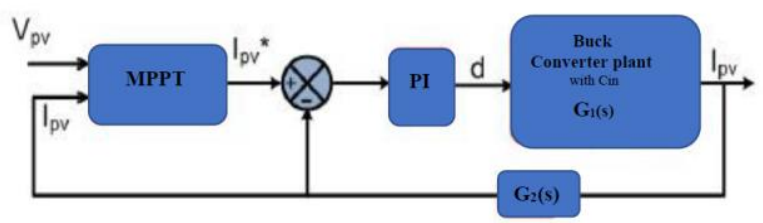

Fig. 2: schematic illustrations of PV powered buck converter including feedback loop [2]

Operating the system in MPPT mode is obligatory over plenty of other photovoltaic powered systems. The inputs $V_{p v}$ and $I_{p v}$ is given to the MPPT controller to generate duty cycle $d$, so that it can transfer maximum from photovoltaic source to a load. The PV fed non ideal buck converter functional block diagram depiction can be seen via Fig.1 accompanied by feedback loop is seen via Fig. 2. In general, the algorithm of maximum power point typically utilizes $V_{p v}$ and $I_{p v}$ as inputs and produces a references called $V_{p v_{r e f}}$ or $I_{p v_{r e f}}$. The value of $V_{p v}$ and $I_{p v}$ should be governed by such references parameters in attempt to transmit the maximum power from photovoltaic source to load. To achieve this , voltage/current reference obtained from MPPT controller is evaluated by real value and perhaps the error will be passed to PI controller so that it produce pulses to a buck converter's switch. From Fig. 2 it is depicted, $G_{1}$ (s) represents the transfer function of buck converter including input capacitor $C_{i n}$. The feedback loop transfer function is represented by $G_{2}(\mathrm{~s})$, which comprises voltage and current sensors and the controller used is PI. A primary and the most step is to derive transfer function of the plant for the design of any controller including P, PI, PID. Depending upon characteristic polynomial of hence derived transfer function of plant, controller poles and zeroes are determined. In this case, buck converter represents the plant including $d$ as controlled input and $i_{p v}$ represents controlled output. As Buck converter will function under continuous and discontinuous conduction modes, thus main intent will be to obtain the transfer function, $\frac{i_{p v}(s)}{d(s)}$ in both cases as well as proving that the transfer function is open loop stable. Therefore in further cases we, initially infer the small signal modelling for buck converter in configuration of state space model representation given by $\dot{X}=A X+B U$ and $Y=C X+$ $D U$ working in CCM mode proceeding by DCM mode , where $X$ represents state variables vector given by $X=$ [ $\left.v_{c_{\text {in }}} i_{L} v_{c}\right], Y$ represents vector of output variables given by $Y=\left[i_{p v}\right]$, U represents the vector of input variables given by $\mathrm{U}=\left[v_{p v} v_{d} d\right]$

\section{A: Continuous Conduction Mode (CCM)}

Equations(1)-(9) represents the algebraic differential equations obtained for non-ideal buck converter displayed in Fig 1 for both the states of switch condition, ON state and OFF state. $\mathrm{R}$ represents the resistive load which is connected at the outlet of the buck converter. From equations (1)-(9), the averaged large signal model is obtained with the duty ratio $d$ and it is linearized by considering the small ac perturbations with the average dc values to develop the small signal models shown by equation (10) and (11). To analyse the impact of maximum power point upon buck converter stability, we will be seeing the percussion influence of $d$ on $i_{p v}$ and hence the transfer function $\frac{i_{p v}(s)}{d(s)}$ is obtained by employing conventional methods from small signal models which is given by equation (12).

ON Condition of the switch:

$$
\begin{aligned}
& \frac{d v_{c_{i n}}}{d t}=\frac{\left(v_{p v}-v_{c_{i n}}\right)}{r_{c_{i n}} c_{i n}} \\
& \frac{d i_{L}}{d t}=\frac{1}{L}\left\{v_{p v}-i_{L}\left(r_{L}+r_{s w}+\frac{R r_{c}}{R+r_{c}}\right)-\frac{R v_{c}}{R+r_{c}}\right\} \\
& \frac{d v_{c}}{d t}=\frac{1}{C}\left\{i_{L}\left(\frac{R}{R+r_{c}}\right)-\frac{v_{c}}{\left(R+r_{c}\right)}\right\} \\
& v_{o}=v_{c}\left(\frac{R}{R+r_{c}}\right)+i_{L}\left(\frac{R r_{c}}{R+r_{c}}\right) \\
& i_{p v}=\frac{v_{p v}-v_{c_{i n}}}{r_{c_{i n}}}+i_{L}
\end{aligned}
$$

OFF Condition of the switch:

$$
\begin{aligned}
& \frac{d v_{c_{i n}}}{d t}=\frac{\left(v_{p v}-v_{c_{i n}}\right)}{r_{c_{i n}} C_{i n}} \\
& \frac{d i_{L}}{d t}=\frac{1}{L}\left\{-i_{L}\left(r_{L}+\frac{R r_{c}}{R+r_{c}}\right)-\frac{R v_{c}}{R+r_{c}}-v_{d}\right\}
\end{aligned}
$$




$$
\begin{aligned}
& \frac{d v_{C}}{d t}=\frac{1}{C}\left\{i_{L}\left(\frac{R}{R+r_{c}}\right)-\frac{v_{C}}{\left(R+r_{C}\right)}\right\} \\
& v_{o}=v_{C}\left(\frac{R}{R+r_{c}}\right)+i_{L}\left(\frac{R r_{C}}{R+r_{C}}\right)
\end{aligned}
$$

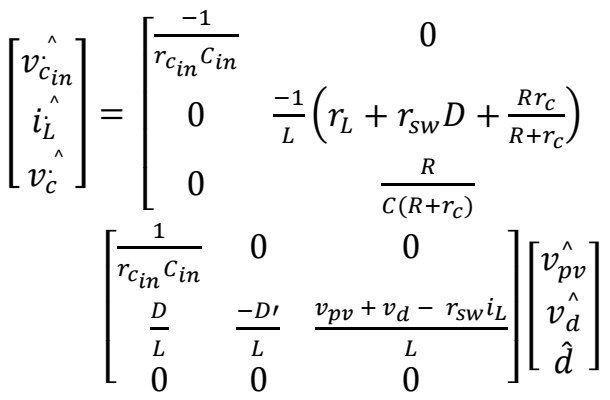$$
\left.\frac{-R}{\frac{-1}{L\left(R+r_{C}\right)}}\right]\left[\begin{array}{c}
v_{c_{i n}} \\
\hat{\imath}_{L} \\
\hat{v}_{c}
\end{array}\right]+
$$

$$
\left[\begin{array}{c}
v_{0}^{\wedge} \\
i_{p v}^{\wedge}
\end{array}\right]=\left[\begin{array}{ccc}
0 & \frac{R r_{c}}{R+r_{c}} & \frac{R}{R+r_{c}} \\
\frac{-1}{r_{c_{i n}}} & 1 & 0
\end{array}\right]\left[\begin{array}{c}
v_{c_{\text {in }}}^{\wedge} \\
\hat{\imath}_{L} \\
\hat{v}_{c}
\end{array}\right]+\left[\begin{array}{ccc}
0 & 0 & 0 \\
\frac{1}{r_{c_{\text {in }}}} & 0 & 0
\end{array}\right]\left[\begin{array}{c}
v_{p v}^{\wedge} \\
\hat{v_{d}} \\
\hat{d}
\end{array}\right]
$$

$$
\frac{i_{p v}(s)}{d(s)}=\frac{\left(s+\frac{1}{r_{c_{i n}} c_{i n}}\right)\left(s+\frac{1}{C\left(R+r_{C}\right)}\right)\left(\frac{v_{p v}+v_{d}-r_{s w} I_{L}}{L}\right)}{\left(s+\frac{1}{r_{c_{i n}} c_{i n}}\right)\left\{\left(s+\frac{1}{L}\left(r_{L}+r_{s w} D+\frac{R r_{C}}{R+r_{c}}\right)\right)\left(s+\frac{1}{C\left(R+r_{C}\right)}\right)+\left(\frac{R}{L\left(R+r_{C}\right)}\right)\left(\frac{R}{C\left(R+r_{c}\right)}\right)\right\}}
$$

\section{B: Discontinuous Conduction Mode (DCM)}

During the discontinuous mode of operation of the buck converter, the differential equations (13)-(26) are obtained for $\mathrm{ON}$ state, $\mathrm{OFF}$ state and the state when the inductor current is zero. These equations are averaged with duty cycles $d_{1}$ and $d_{2}$. $d_{1}$ represents the ratio of ON time of the buck converter switch to the total switching period. $d_{2}$ represents the ratio of the inductor current when it returns to zero point during the OFF time of switch of the converter to the Ts (total switching period). The averaged model containing $d_{1}$ and $d_{2}$ should now be modified to eliminate $d_{2}$ [5].

\section{ON Condition of the switch}

$\frac{d v_{c_{i n}}}{d t}=\frac{\left(v_{p v}-v_{c_{i n}}\right)}{r_{c_{i n}} C_{i n}}$

$\frac{d i_{L}}{d t}=\frac{1}{L}\left\{v_{p v}-i_{L}\left(r_{L}+r_{s w}+\frac{R r_{C}}{R+r_{c}}\right)-\frac{R v_{C}}{R+r_{c}}\right\}$

$\frac{d v_{c}}{d t}=\frac{1}{C}\left\{i_{L}\left(\frac{R}{R+r_{C}}\right)-\frac{v_{c}}{\left(R+r_{C}\right)}\right\}$

$v_{o}=v_{c}\left(\frac{R}{R+r_{c}}\right)+i_{L}\left(\frac{R r_{C}}{R+r_{c}}\right)$

$i_{p v}=\frac{v_{p v}-v_{c_{i n}}}{r_{c_{\text {in }}}}+i_{L}$

OFF condition of the switch

$$
\begin{aligned}
& \frac{d v_{c_{i n}}}{d t}=\frac{\left(v_{p v}-v_{c_{i n}}\right)}{r_{c_{i n}} C_{i n}} \\
& \frac{d i_{L}}{d t}=\frac{1}{L}\left\{-i_{L}\left(r_{L}+\frac{R r_{C}}{R+r_{c}}\right)-\frac{R v_{C}}{R+r_{c}}-v_{d}\right\} \\
& \frac{d v_{C}}{d t}=\frac{1}{C}\left\{i_{L}\left(\frac{R}{R+r_{c}}\right)-\frac{v_{C}}{\left(R+r_{c}\right)}\right\} \\
& v_{o}=v_{c}\left(\frac{R}{R+r_{C}}\right)+i_{L}\left(\frac{R r_{C}}{R+r_{c}}\right)
\end{aligned}
$$

During $\left(1-d_{1}-d_{2}\right) T_{s}:$ When the inductor current is zero

$\frac{d v_{c_{i n}}}{d t}=\frac{\left(v_{p v}-v_{c_{i n}}\right)}{r_{c_{i n}} C_{i n}}$

$\frac{d i_{L}}{d t}=0$

$\frac{d v_{c}}{d t}=\frac{1}{C}\left\{-\frac{v_{c}}{\left(R+r_{C}\right)}\right\}$

$$
i_{p v}=\frac{v_{p v}-v_{c_{i n}}}{r_{c_{\text {in }}}}
$$

$v_{o}=v_{c}\left(\frac{R}{R+r_{c}}\right)$

Average inductor current of the buck converter is given by : $i_{L}=\frac{1}{2} i_{p k}\left(d_{1}+d_{2}\right)$

Where $i_{p k}$ represents the maximum inductor current, $i_{p k}=\frac{\left(v_{p v}-i_{L}\left(r_{L}+r_{S W}\right)-v_{o}\right) d_{1} T_{S}}{L}$ substituting $i_{p k}$ in equation (27) we obtain,

$d_{2}=\frac{2 L i_{L}}{\left(v_{p v}-i_{L}\left(r_{L}+r_{S w}\right)-v_{o}\right) d_{1} T_{S}}-d_{1}$

Here $T_{S}$ represents the total switching period and it is given by $T_{s}=\frac{1}{f_{s}}$ and $f_{s}$ represents the switching frequency of the buck converter. From equation (28) which demonstrates $d_{2}$ as a function of $d_{1}$ has been further simply replaced in an averaged model to develop a model independent of $d_{2}$. Therefore the model hence derived is linearised to develop the small signal models depicted by equations (29) and (30) and the co-efficient of the model is depicted in appendix. The transfer function for DCM is given by equation (31)

$$
\begin{aligned}
& {\left[\begin{array}{c}
v_{c_{i n}}^{\wedge} \\
i_{\dot{L}}^{\wedge} \\
v_{c}^{\wedge}
\end{array}\right]=\left[\begin{array}{lll}
P_{11} & P_{12} & P_{13} \\
P_{21} & P_{22} & P_{23} \\
P_{31} & P_{32} & P_{33}
\end{array}\right]\left[\begin{array}{c}
v_{c_{i n}}^{\wedge} \\
\hat{i_{L}} \\
\hat{v}_{c}
\end{array}\right]+\left[\begin{array}{lll}
Q_{11} & Q_{12} & Q_{13} \\
Q_{21} & Q_{22} & Q_{23} \\
Q_{31} & Q_{32} & Q_{33}
\end{array}\right]\left[\begin{array}{c}
v_{p v} \\
v_{d} \\
\hat{d}
\end{array}\right]} \\
& {\left[\begin{array}{c}
v_{0}^{\wedge} \\
i_{p v}^{\wedge}
\end{array}\right]=\left[\begin{array}{lll}
W_{11} & W_{12} & W_{13} \\
W_{21} & W_{22} & W_{23}
\end{array}\right]\left[\begin{array}{c}
v_{c_{i n}} \\
\hat{\imath}_{L} \\
\hat{v}_{c}
\end{array}\right]+\left[\begin{array}{lll}
S_{11} & S_{12} & S_{13} \\
S_{21} & S_{22} & S_{23}
\end{array}\right]\left[\begin{array}{c}
v_{p v}^{\wedge} \\
v_{d} \\
\hat{d}
\end{array}\right]}
\end{aligned}
$$

$$
\frac{i_{p v}(s)}{d(s)}=\frac{Q_{23}\left(s-P_{11}\right)\left(s-P_{33}\right)}{\left(s-P_{11}\right)\left(\left(s-P_{22}\right)\left(s-P_{33}\right)-P_{33} P_{23}\right.}
$$




\section{C: Observations}

The extrapolations from contrast (12) and (31) are as follows

1. From both CCM and DCM cases, has pole - zero cancellation located close to $s=-\frac{1}{r_{c_{i n}} c_{i n}}$. Because neither of the other expressions comprises $r_{c_{i n}} \quad$ and $\quad C_{i n}$, hence stability is autonomous of input capacitor.

2. In each of these CCM and DCM cases, the zero resides in Left Hand side of the Plane (LHP) which is a function of $r_{c}$, Load, Output Capacitance.

3. Both for CCM and DCM cases, the characteristic equation includes all positive coefficients and as per the RH criteria (Routh-Hurwitz) for second order system, system is stable under all conditions.

The observations derived above are not only true for $\frac{i_{p v}(s)}{d(s)}$, this could also be shown by detailed derivations to obtain the transfer function for $\frac{v_{p v}(s)}{d(s)}$, similar observations could be obtained. Thus it can be concluded that even for PV fed buck converter which is working under both CCM and DCM modes, the stability of MPPT system is not dependant on input capacitance and its esr and also it is open loop stable. The investigation which is carried out for boost converter in recent research holds good for buck converter giving the similar observations.

\section{Simulation Results}

Designed parameters are given by, a PV Panel with $P_{M P P, \text { ref }}=$ $74.5 \mathrm{~W}, I_{M P P, \text { ref }}=4.4 \mathrm{~A}, V_{M P P, \text { ref }}=17 \mathrm{~V}$. A buck converter with $C_{\text {in }}=2000 \mu \mathrm{F}, r_{\text {cin }}=0.016 \Omega, L=1.35 \mathrm{mH}, \mathrm{r}_{\mathrm{L}}=0.7 \Omega, \mathrm{r}_{\mathrm{sw}}=50 \mathrm{~m}$ $\Omega, \mathrm{v}_{\mathrm{d}}=1.65 \mathrm{~V}, \quad C=1000 \mu \mathrm{F}, f_{s}=25 \mathrm{kHz}, \mathrm{r}_{\mathrm{c}}=0.032 \Omega$. Вy substituting these values in the derived transfer function $\frac{i_{p v}(s)}{d(s)}$ , which is obtained from small signal models for both CCM and DCM case, we generate a bode plot using MATLAB Simulink. To illustrate the buck converter's (plant ) open loop stability, the bode plots for transfer function in both the cases are represented in Fig 3 and Fig 4 respectively. The PV system is assumed to be run in constant current mode condition with $i_{p v}=4 \mathrm{~A}$ and $\mathrm{R}=20 \Omega$ in Continuous Current Mode operation and the results are displayed. It is observed that gain margin (GM) is shown to be infinite and phase margin (PM) is equivalent to $92.3^{\circ}$ implying that system is robust and stable. Similarly in DCM condition the bode plot is plotted for $i_{p v}=0.5 \mathrm{~A}$ and load with $\mathrm{R}=2000 \Omega$ , illustrating that the GM margin is infinite and PM is near to $92.38^{\circ}$ proving that the system is open loop stable.

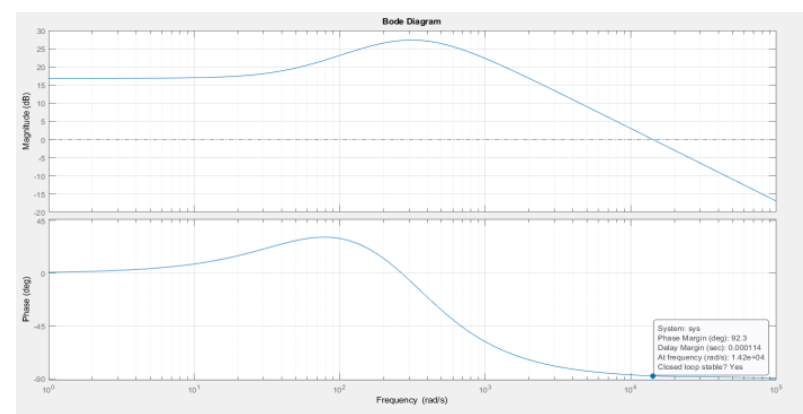

Fig.4 : Bode Plots demonstrating open loop stability under constant current mode in CCM case

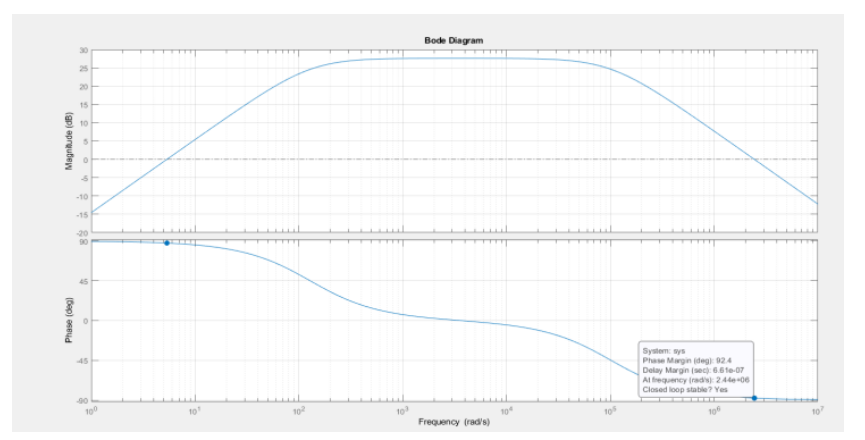

Fig.4 : Bode plots demonstrating open loop stability under constant current mode in DCM case.

\section{CONCLUSIONS}

In this paper, the investigation is carried out for PV powered non - ideal buck converter by researching the impact of additional capacitor at the photovoltaic system terminals on the system stability is been analyzed by the detailed derivations of small signal models. This investigation gave the similar conclusions from the recent research for PV fed boost converter. Stability models for other power converters were not derived yet. Including the non - idealities of the parameters the small signal models are obtained in both the operating modes, CCM and DCM. Through these models, transfer function is derived for both the operating modes which comprises an identical pair of pole and zero, which are the functions of input capacitor and its effective series resistance. It has also inferred by pole - zero cancellations that system stability is not dependent on input capacitor and esr. RH criteria shows that system is stable when all the coefficient are positive, and all poles and zeros lies in LHP of s plane indicating that system is open loop stable.

\section{FUTURE SCOPE}

This investigation can be performed for other power converters as well for the analysis of impact of introduction of input capacitor upon stability of system through small signal modelling.

\section{APPENDIX}

$P_{11}=\frac{-1}{r_{c_{i n}} C_{i n}} ; \quad P_{33}=\frac{-1}{C\left(R+r_{c}\right)} ;$ 


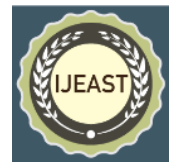

$P_{12}=P_{13}=P_{21}=P_{31}=0$;

$P_{23}=-\frac{2 I_{L} \frac{R}{R+r_{C}}\left\{v_{d+} v_{p v}-I_{L}\left(r_{L}+r_{S w}+\frac{R r_{C}}{R+r_{C}}\right)\right\}}{d_{1} T_{S}\left(v_{p v}-I_{L}\left(r_{L}+r_{S w}+\frac{R r_{C}}{R+r_{c}}\right)-\frac{R v_{C}}{R+r_{C}}\right)^{2}}+\frac{d_{1}^{2} T_{S} r_{S W} R}{2 L^{2}\left(R+r_{C}\right)} ;$

$P_{32}=\frac{R}{C\left(R+r_{C}\right)} \quad ;$

$P_{22}=\frac{-1}{2 L^{2}\left(R+r_{c}\right)}\left\{2 L\left[r_{L}\left(R+r_{c}\right)+R r_{c}\right]+d_{1}^{2} T_{s}\left[-\left(r_{L}+\right.\right.\right.$

$\left.\left.\left.r_{s w}+\frac{R r_{c}}{R+r_{c}}\right) r_{s w}\left(R+r_{c}\right)\right]\right\}-\left(\frac{2 R v_{c}}{R+r_{c}}+\right.$

$\left.2 v_{d}\right)\left(\frac{v_{p v}-\frac{R v_{C}}{R+r_{C}}}{d_{1} T_{S}\left[v_{p v}-I_{L}\left(r_{L}+r_{S w}+\frac{R r_{C}}{R+r_{C}}\right)-\frac{R v_{C}}{R+r_{C}}\right]^{2}}\right)$;

$Q_{11}=\frac{1}{r_{c_{\text {in }}} C_{\text {in }}} ; Q_{12}=Q_{13}=Q_{31}=Q_{32}=Q_{33}=0$;

$Q_{21}=-\frac{d_{1}^{2} T_{S} r_{S w}}{2 L^{2}}+\frac{d_{1}}{L}+\frac{2 I_{L}\left(\frac{R v_{C}}{R+r_{C}}+v_{d}\right)}{d_{1} T_{S}\left(v_{p v}-I_{L}\left(r_{L}+r_{S w}+\frac{R r_{C}}{R+r_{C}}\right)-\frac{R v_{C}}{R+r_{C}}\right)^{2}} ;$

$Q_{22}=\frac{-1}{L}\left\{\frac{2 L I_{L}}{d_{1} T_{S}\left(v_{p v}-I_{L}\left(r_{L}+r_{S W}+\frac{R r_{C}}{R+r_{C}}\right)-\frac{R v_{C}}{R+r_{C}}\right)}-d_{1}\right\} ;$

$Q_{23}=-\frac{d_{1} T_{s}}{L^{2}}\left[v_{p v}-I_{L}\left(r_{L}+r_{s w}+\frac{R r_{c}}{R+r_{c}}\right)-\frac{R v_{c}}{R+r_{c}}\right] r_{s w}+\frac{v_{p v}}{L}+$

$\frac{v_{d}}{L}+\left(\frac{v_{d}}{L}+\frac{R v_{c}}{L\left(R+r_{c}\right)}\right)\left\{\frac{2 L I_{L}}{d_{1}^{2} T_{S}\left(v_{p v}-I_{L}\left(r_{L}+r_{S w}+\frac{R r_{C}}{R+r_{c}}\right)-\frac{R v_{C}}{R+r_{C}}\right)}\right\}$;

$S_{11}=S_{12}=S_{13}=S_{22}=S_{23}=0 ; E_{21}=\frac{1}{r_{c_{\text {in }}}}$;

$W_{11}=W_{23}=0 ; W_{22}=1$;

$W_{12}=\frac{R r_{c}}{R+r_{c}} ; W_{13}=\frac{R}{R+r_{c}} ; W_{21}=-\frac{1}{r_{c_{i n}}} ;$

\section{REFERENCES}

[1] Tsung-Wei Hsu, Hung-Hsien Wu, Dian-Lin Tsai and Chia-Ling, Wei, "Photovoltaic Energy Harvester with Fractional Open-Circuit Voltage Based Maximum Power Tracking Circuit", IEEE Trans. Circuits Syst.II, Express Briefs, DOI 10.1109/TCASII.2018.2838672.

[2] Efstratios I. Batzelis, Georgious Anagnostou and Bikash C. Pal,July 2018,“A State- Space Representation of Irradiance-Driven Dynamics in Two-Stage Photovoltaic Systems", IEEE J. Photovolt.,Vol 8, No. 04.
[3] Erickson, Robert W., and Dragan Maksimovic, 2007 "Fundamentals of Power Electronics, Springer Science and Business Media.

[4] Pallavi Bharadwaj, Vinod John, June 2015 "Direct Duty Ratio Controlled MPPT Algorithm for Boost converter in Continuous and Discontinuous Modes of Operatiom", IEEE 6th India International Conference on Power Electronics, Kurukshetra, India.

[5] Jian Sun, Daniel M Mitchell, Matthew F. Greuel, Philip T. Krien and Richard M. Bass, July 2001, "Averaged Modeling of PWM Converters Operating on Discontinuous conduction mode", IEEE Transactions on Power Electronics, Vol 16, No. 04.

[6] Arjun M, Vanjari Venkata Ramana, Roopa Viswadev, B Venkatesaperumal,2018, "Small Signal Model for PV fed Boost Converter in Continuous and Discontinuous Conduction Modes", IEEE Transactions on Circuits and Systems II: Express Briefs.

[7] Diego R. Espinoza-Trejo, Ernesto Barcenas-Barcenas, Daniel U. Campos-Delgado and Cristian H. De Angelo, June 2015, "Voltage-Oriented Input-Output Linearization Controller as Maximum Power Point Tracking Technique for Photovoltaic Systems", IEEE Trans. Ind. Electron.,, Vol.62, No. 06.

[8] Hongda Cai, Ji Xiang, Wei Wei, June 2016 "Modelling, analysis and control designof a two-stage photovoltaic generation system", IET Renew. Pow. Gener,Vol 10, Iss. 8.

[9] Nicola Femia, Gianpaolo Lisi, Giovanni Petrone, Giovanni Spaguoloand Massimo Vitelli, July 2008, "Distributed Maximum Power Point Tracking of Photovoltaic Arrays: Novel Approach and System Analysis", IEEE Transactions on Industrial Electronics, Vol 55, No. 07.

[10] Weichao Zhang and Jonathan W. Kimball, July 2018 "DC-DC Converter Based PV simulator with a double Current Mode Controller", IEEE Transactions on Power Electronics, Vol 33.

[11] M. G. Millalva, T.G. de Siqueira and E. Ruppert, Dec 2010 "Voltage Regulation of Photovoltaic arrays: Small signal analysis and control design", IET Power Electronics, Vol. 03.

[12] Ling Qin, Shaojun Xie, Mao Hu, Chen Yang, May 2015 "Stable operating area of photovoltaic cells feeding DC-DC converter in output voltage regulation mode", IET Renewable Power Generation, Vol. 09.

[13] Eugene V. solodovnik, Shengyi Liu and Roger A. Dougal, Sep 2004 "Power Controller Design for Maximum Power Tracking in Solar Installations" IEEE Transactions on Power Electronics, Vol 19, No. 05. 